\title{
OTHONOMICS
}

Revista de economía, empresa y sociedad

Dosier "Vectores de sostenibilidad: visiones desde la economía»

Coordinador: Albert Puig Gómez

UNA PERSPECTIVA HISTÓRICA

\section{La geopolítica de las renovables en el capitalismo del siglo XXI}

\section{Aurèlia Mañé-Fstrada}

Universitat de Barcelona

RESUMEN El artículo analiza la geopolítica de la energía en el marco del sistema capitalista desde una perspectiva histórica. La primera sección explica el nacimiento de la geopolítica de la energía (una determinada geografía de la energía y relaciones entre los Estados) y argumenta que este es un hecho asociado con la energía fósil y que se fundamenta en el mantenimiento de la hegemoníaa causa de control territorial de las fuentes energéticas (o flujos energéticos) y su mercantilización. Después de un breve repaso por la geopolítica del petróleo, el artículo especula sobre cuál podría ser la geopolítica de las renovables en el capitalismo del siglo XXI. La principal conclusión es que, debido a las características intrínsecas de las fuentes renovables, el tipo de geopolítica que se establezca será el fruto de una elección. La cuestión es si esta elección irá encaminada también a utilizar las relaciones energéticas internacionales como sustrato para la hegemonía mundial y la financiación del sistema.

PALABRAS CLAVE transición energética; geopolítica de las renovables; capitalismo; economía política; historia de la energía

\section{A HISTORICAL PERSPECTIVE}

\section{The geopolitics of renewables within 21 st century capitalism}

\begin{abstract}
The article analyses, from a historical perspective the geopolitics of energy in the framework of the capitalist system. In the first section, it explains the birth of the geopolitics of energy (a stated geography of energy and the kind of energy relations between states), arguing that its birth is associated with fossil fuels and its goal is the safeguarding of supremacy through both territorial control of energy sources (or flows) and their commodification. After a brief review of the geopolitics of oil, the article ventures on what, at the present juncture of capitalism, the geopolitics of renewables might be. The main conclusion is that, because of inherent features of renewables, at the end of the day the outcome will be the result of a political choice. The question is whether this choice will also be aimed at both grounding hegemony on international energy relations and funding the global unbalances of the system.
\end{abstract}

KEYWORDS energy transition; geopolitics of energy; capitalism; political economy, energy history 


\section{Introducción}

La cuestión de la geopolítica de las renovables se ha convertido en una de las cuestiones protagonistas del debate sobre la transición energética. Esta geopolítica se contrapone a la del petróleo o, en sentido más amplio, a la de las energías fósiles. De hecho, lo que este debate plantea es si un cambio en las fuentes energéticas significará un cambio en las relaciones políticas entre países o regiones, debido a un cambio en la geografía de la energía o de un cambio en la localización de las fuentes energéticas.

\section{Fundamentos básicos de la geopolítica de la energía}

Una de las principales diferencias entre las energías fósiles y lo que genéricamente denominamos renovables (fuentes energéticas orgánicas, como la biomasa, o no orgánicas, como el viento, el sol o las corrientes fluviales o marinas) es que las primeras (carbón, petróleo, gas y, por asimilación, el uranio) solo se hallan en determinados territorios, mientras que las segundas, en combinaciones distintas (mix energético), se encuentran por todas partes. Por este motivo, en todos los países del mundo, de una forma u otra, es posible generar energía útil para los seres humanos a partir de fuentes renovables. En este sentido, es interesante señalar que los historiadores de la energía (Malanima, 2014) apuntan el hecho de que, en el mundo, hasta mediados de siglo XIX, con el advenimiento de la Revolución Industrial y la adopción del carbón como fuente energética hegemónica del sistema capitalista, no hubo desigualdades fundamentales entre territorios por causa energética.

Es con la Revolución Industrial (Wrigley, 2010; Warde, 2013) y con la instauración del capitalismo manufacturero, cuando, debido a la combinación entre la máquina de vapor y el carbón, la Inglaterra victoriana se convirtió en la primera potencia del mundo.

Esta hegemonía, la de un país por encima de los demás, justificada por el factor territorial de que en el interior de unas fronteras (que después se extenderán, por el control de concesiones mineras en territorios de ultramar) la Providencia hubiere ubicado recursos energéticos, es uno de los principales argumentos del libro The Coal Question, de 1865, de William Stanley Jevons, uno de los padres de la economía matemática marginalista.

Este año, este libro se ha vuelto icónico entre los economistas del decrecimiento, por la descripción que el autor hace de lo que se conoce como la paradoja de Jevons. Más allá de esta, The Coal Question es el fundamento de la moderna geopolítica de la energía, pues en el libro se reflejan tres cuestiones. En primer lugar, que, según el autor, la supremacía -moral, intelectual y material- del Reino Unido por encima del resto de las naciones del mundo se debe a la existencia en suelo propio de carbón abundante, de buena calidad y de coste -relativamente- más barato que el del resto del mundo. En segundo lugar, que esta hegemonía se tambalearía si hubiera en el mundo (como en la época ocurrió por el carbón de Estados Unidos) otros territorios que tuvieran más carbón, mejor y más barato. Y, por último, el libro se hace eco del pensamiento imperial británico de la época, al asumir que las renovables no son una alternativa políticamente viable al carbón, a pesar de que aunque sí que podrían ser una opción energética. No lo son en términos de hegemonía. Como queda recogido en el libro, aunque en el Reino Unido hay viento, ríos y mareas, fuentes también susceptibles de generar energía motriz, estas no son alternativas viables al carbón, dado que [...] «these are common to all the world».

A este argumento, que se fundamenta en la lógica del poder, se suma otro basado en la lógica de la propiedad y la ganancia. Sobre unos terrenos donde hay minas de carbón, como también sobre aquellos en los que hay yacimientos de petróleo o gas y minas, se pueden establecer derechos de propiedad. Estos son la base para el comercio de la mercancía (toneladas de carbón, barril de petróleo, metro cúbico de gas o pastilla de uranio) extraída en este lugar. Por ello, la segunda característica del modelo de energía fósil es que se puede «hacer negocio» con la compraventa de las fuentes energéticas, puesto que el propietario del terreno, o a quien se le hayan otorgado los derechos de su explotación, disfrutará de la potestad de decidir a quién vende este producto y en qué condiciones. Obviamente, esta posibilidad no existe en fuentes energéticas como el viento o el sol; su condición de bienes libres no los hace susceptibles ni de propiedad -excluyente del otro- ni de intercambio mercantil. 
Un último aspecto que se ha de tener en cuenta es que las fuentes fósiles y minerales, como todo producto escaso de la tierra, son susceptibles de generar renta -ricardiana- si existe un mercado unificado. Por ejemplo, en el caso del petróleo, por existir «el precio internacional», se paga al mismo precio el petróleo de Oriente Medio, con unos costes de extracción muy bajos, que el de las arenas bituminosas de Canadá, donde estos mismos costes son muy elevados. Así, los propietarios de los yacimientos de Oriente Medio -o quien tenga otorgada la concesión de su producto-, cuando su petróleo se venda a un precio que permita cubrir los gastos y obtener beneficios, en Canadá, disfrutarán de unos ingresos «adicionales», que es la renta del petróleo. Obviamente, cuando los bienes son libres y abundantes, como el sol o el aire, es imposible obtener renta.

Siguiendo el sentido de lo escrito hasta ahora, cabe señalar que, para que la geopolítica de las renovables tuviera unas características similares a las que en el capitalismo ha tenido la de la energía fósil -poder político territorial desigual fundado en una distribución geográfica desigual de las fuentes energéticas, negocio privativo y excluyente de las fuentes energéticas, y obtención de rentas mineras a causa de la creación de un mercado internacional del petróleo unificado, en argot, el great pool del petróleo-, se tendrían que crear artificialmente unas condiciones equivalentes de desigualdad y poder territorial, de propiedad y de rentas, por medio del control de las patentes sobre la tecnología y de la propiedad de las infraestructuras energéticas.

\section{La geopolítica de la energía en el siglo XX}

La geopolítica del petróleo durante los años posteriores a la Primera Guerra Mundial se articuló alrededor de unos tratados y acuerdos, resultado de una lógica imperial británica y de otra empresarial norteamericana, que fijaron la geografía contemporánea de la energía. Desde entonces, en el marco de la División Internacional del Trabajo (DIT) capitalista, el petróleo de la región de Oriente Medio es el eje en torno al cual se articula la geopolítica del petróleo, así como la arquitectura de la estructura de gobernanza de la industria petrolera internacional. La función de esta estructura es establecer un precio, una variable económica, que es el resultado del juego de poder entre los Gobiernos de los principales países productores, los de los países consumidores y los grandes consorcios petroleros transnacionales de compañías privadas y estatales.

En el sistema capitalista, este precio, además de ser el que se paga por la unidad «barril de petróleo», tiene dos funciones, adicionales, que son las relevantes para los actores que interactúan en el juego -de poder- petrolero. La primera es lograr que el precio de la energía, como factor de producción, sea el que más convenga a las necesidades geopolíticas de la economía hegemónica (en función de los momentos y, hasta ahora, la de Estados Unidos o las de la OCDE); y, la segunda, conseguir que este precio, tal como hemos explicado en el apartado anterior, permita una generación de renta «apropiada» para otorgar una renta nacional «suficiente» a los países productores y unos beneficios «adecuados» a las compañías petroleras internacionales. Además, desde la década de 1970, debido a las necesidades crecientes de la financiación de la economía estadounidense, el precio del petróleo (o la renta que se genera a partir de este precio) tiene que permitir generar un excedente suficiente como para financiar las economías nacionales deficitarias mediante el reciclaje de petrodólares, fundamentalmente por medio de la City de Londres y del mercado de eurodólares.

Este último aspecto es el que inspira lo que se podría denominar como el pacto petrolero de los años setenta; el que se forjó alrededor de los llamados shocks del petróleo. Este es un pacto -explícito o tácito, según las fuentes- que otorga a la Organización de los Países Exportadores de Petróleo (OPEP) la función de velar por el precio «adecuado", para el cumplimiento de las dos funciones mencionadas: ayudar a la competitividad de la economía hegemónica y la generación de una renta "suficiente». A cambio, de acuerdo con las resoluciones de Naciones Unidas que reconocieron el derecho de los Estados -entonces, recientemente descolonizados y del tercer mundo- a la soberanía permanente sobre los recursos naturales ubicados en su territorio,' se aceptaron las nacionalizaciones de los activos que las compañías petroleras internacionales tenían en los territorios nacionales de los países y de la renta que su explotación generaba, siempre y cuando esta se gastara o se invirtiera en las economías de los países consumidores.

1. En lo referente a esto, ved la resolución 1803 (XVII) de la Asamblea General de las Naciones Unidas, 1962. 
Con todo ello, este orden petrolero otorgó una peculiar forma a la geopolítica asimétrica de la energía entre dos categorías de Estados, las de los países consumidores, de la OCDE, y los productores. En esta geopolítica, por delegación, la función de la superestructura de estos últimos, la OPEP (de hecho, la de Arabia Saudí actuando como swing producer), por delegación, era la de ayudar a la regulación del precio del petróleo internacional, para que este cumpliera adecuadamente con sus funciones.

Lo que hemos visto hasta este momento nos permite abordar la geopolítica de las renovables desde dos puntos de vista:

a) el de las circunstancias geográficas de las fuentes energéticas -localización territorial desigual apropiable, sobre la cual se puede fundamentar la diferencia entre economías y países- , y

b) el de la función del precio, como precio del factor de producción de energía, que ayuda a mantener la hegemonía y el de la renta, que estas fuentes generan, como excedente para financiar los desequilibrios del sistema capitalista.

En la práctica, los principales elementos que han condicionado la geopolítica de la energía fósil a lo largo del siglo XX han sido la voluntad del poder hegemónico mundial del control, directo o indirecto, de estos recursos para sostener su supremacía, y la creación de una superestructura internacional para la gestión del precio-renta del petróleo. Ello es lo que ha otorgado una cierta capacidad de influencia a las denominadas economías rentistas, especialmente las petromonarquías del golfo Pérsico y, en función de los momentos y en un grado menor, países como Argelia, Iraq, Siria, Libia o Venezuela.

\section{La geopolítica de las renovables en el siglo XXI}

Por lo que se ha explicado en la primera sección, ya queda claro que las características intrínsecas de las fuentes renovables -encontrarse por todas partes, ser libres y abundantes, o no agotables- favorecerían que no hubiera una distribución territorial desigual del poder debido a una distribución geográfica desigual de las fuentes energéticas. También, por el hecho de ser, estas, unas fuentes libres y abundantes, no tendría que haber ninguna mercantilización (o negocio basado en estas). Por ello, económicamente, no existe ninguna razón que justifique un precio del sol o del aire. Por la misma razón, no habría posibilidad de generación de renta a partir de estas fuentes renovables.

Así, sobre el papel, una transición hacia un modelo de generación de energía útil para los humanos, basada en energías renovables, pondría fin a la necesidad del sistema de fundar la hegemonía de determinadas economías o Estados en el control -territorial- de un recurso natural, ya que no sería posible el precio del factor productivo energía de forma que fuera favorable a sus intereses, como también sería imposible generar excedentes susceptibles de financiar los desequilibrios económicos globales.

Desde este punto de vista, la transición energética hacia un modelo basado en renovables sería, casi, revolucionaria, puesto que a la vez podría significar, a la vez, el fin de la supremacía de unos territorios por encima de los otros por tener un «mejor» acceso a las fuentes energéticas, y también pondría fin a los excedentes financieros que sostienen los desequilibrios económicos (y sociales) fundamentales del sistema.

A pesar de ello, hay dos elementos adicionales que hay que tener en cuenta a la hora de analizar esta cuestión. El primero es que las fuentes energéticas necesitan ser transformadas y distribuidas de alguna manera para que los humanos podamos utilizar la energía. El segundo es que los «modelos» energéticos no se desarrollan en el vacío, sino en el marco de un sistema con sus relaciones de poder.

\subsection{Dos posibles sistemas de energía renovable}

Cuando hablamos de fuentes renovables (cosa que es más difícil con las fósiles), podemos elegir entre dos modelos; uno que apostaría más por sistemas energéticos de «proximidad» con infraestructuras de tamaño pequeño o mediano, gestionados -en varias modalidades- por pequeñas y medianas empresas locales, que se escalarían regional y nacionalmente por medio de redes descentralizadas y distribuidas. El modelo alternativo es el que pretende replicar la estructura actual del modelo fósil, creando una nueva geografía de países desde los cuales se exportaría la energía hacia otras donde se importaría y se utilizaría. Esta segunda opción remite a infraestructuras transnacionales colosales, gestionadas por grandes consorcios empresariales, que, de manera centralizada (como ahora), incidirían sobre las condiciones de acceso a la red. 
En el momento actual, ambas propuestas están presentes en el debate energético.

La primera es una realidad de hecho en determinadas zonas de Alemania, y se conoce como Energiewende. Si esta se generalizara a escala planetaria ocasionaría un giro copernicano a la geopolítica mundial de la energía. En el caso de su (poco probable) universalización, desaparecería la necesidad de relación entre Estados por causa del suministro de fuentes energéticas: si la transición se cumpliera plenamente, desaparecería el comercio (y el negocio) internacional de energía tal y como lo hemos conocido hasta ahora.

Sin embargo, si hoy en día miramos qué opciones de generación y comercialización de energía renovable hay sobre la tabla, veremos la proliferación de iniciativas que buscan convertir las relaciones energéticas renovables en algo similar a las de la energía fósil.

Entre estas, se contraponen dos grupos de propuestas: ${ }^{2}$

a) las de red, que, de hecho, deslocalizan la generación de electricidad a espacios poco poblados y alejados, como desiertos, para el caso de la energía solar, o de alta mar, para el caso de la energía eólica, desde los que conectar unos cables de altísima tensión que, después de cruzar mares y fronteras, transportarán la electricidad generada en los países productores hacia los consumidores.

b) las de generación de hidrógeno en el tercer mundo, en lugares como la Tierra del Fuego o Marruecos, que se exportaría hacia el Norte global con infraestructuras similares a las del gas vía hidrogenoductos o barcos.

Aunque ambas propuestas son de cariz diferente, e impulsadas por actores económicos diferentes -los grandes grupos eléctricos y de infraestructuras, la primera, y sectores como el automovilístico alemán y del gas europeo, la segunda-, las dos pretenden mantener la actual dicotomía entre país exportador e importador, conectados por medio de infraestructuras transnacionales.

Al ser parecidos, justo es decir que, a diferencia del modelo fósil, la dicotomía, para el caso de las renovables, es una elección que no se justifica por la existencia de una distribución desigual de los recursos, sino por una creada voluntariamente trazando y conectando infraestructuras, que redunda en una desigualdad territorial energética que no existía previamente.

Ahora bien, a pesar de que la geopolítica de las renovables pudiera tener similitudes con la de la energía fósil, merece la pena señalar dos de sus principales diferencias.

La primera es que las renovables abren la puerta a una geopolítica de diseño, puesto que la nueva relación territorial sería fruto de una doble elección política: decidir deslocalizar la generación y elegir el país donde hacerlo.

La segunda es que las renovables abren la puerta a finalizar con la geopolítica energética tradicional, la que se lleva a cabo entre Estados. La razón fundamental reside en las características intrínsecas de las fuentes renovables: hallarse en todas partes, ser bienes libres, inagotables y abundantes. En términos de poder de los Estados, esto implica la imposibilidad, por no identificarse con ningún territorio ni ser apropiables, de estar sujetos a las resoluciones que aseguran la soberanía de los Estados sobre los recursos que se hallan en su territorio (económicamente, es muy diferente alquilar un terreno que otorgar una concesión para la explotación de un recurso nacional sobre el terreno).

Desde este punto de vista, es probable que aumente, todavía más, el peso internacional de las empresas propietarias de las infraestructuras, frente a los Estados como custodios de los recursos nacionales. También, hay que decirlo, porque la lógica de suministro nacional de las políticas energéticas de los territorios importadores se ha ido desdibujando desde la década de 1980.

\subsection{La energía renovable en el sistema capitalista contemporáneo}

Si bien, como acabamos de ver, el control territorial de los flujos energéticos renovables se puede reproducir artificialmente mediante la construcción de infraestructuras transnacionales, las diferencias señaladas plantean un tema espinoso para el funcionamiento actual del sistema capitalista.

La imposibilidad de control nacional de los recursos renovables y la imposibilidad de un comercio de fuentes renovables (sol, aire, corrientes marinas o fluviales...) imposibilita que de manera «razonable» pueda haber un mercado (o precio) internacional, como el que existe para el petróleo, y, por la misma razón, no es factible la obtención de renta a partir de fuentes renovables universales, libres, inagotables y abundantes.

2. Muestras de estos proyectos se pueden consultar en la web de IRENA (https://www.irena.org/). 
En un sistema como el nuestro, altamente financiarizado y que se mantiene gracias a la existencia de flujos que alimentan los desequilibrios globales entre economías endeudadas, como Estados Unidos, y excedentarias, como Arabia Saudí, el abandono progresivo de las energías fósiles obligaría a replantear el funcionamiento global del sistema. Es cierto que se limitaría mucho el poder que países rentistas, como Qatar, han adquirido gracias a su pujanza financiera, pero también es cierto que buena parte de las economías del Norte global, como Estados Unidos, el Reino Unido o España, dejarían de recibir los flujos financieros que las financian.

Ante esto, podría incurrir en dos tentaciones para mantener la hegemonía actual. La primera, del mismo modo que se podría caer en una geopolítica de diseño, se podría caer en la de renta energética «ficticia»: aquella resultado del monopolio de los consorcios energéticos transnacionales que controlarían los flujos eléctricos y de hidrógeno. La segunda sería buscar nuevas fuentes de renta, con la emergencia de una nueva geopolítica de los minerales raros. Minerales que, en muchos ocasiones, se asocian con las nuevas formas de uso de la energía y las nuevas tecnologías asociadas.

\section{Conclusiones}

En la introducción, nos preguntábamos si un cambio en las fuentes energéticas comportaría una modificación en las relaciones políticas entre países o regiones, por producirse un cambio en la geografía de la energía. Después de todo lo que se ha argumentado, la respuesta a esta pregunta no es evidente, puesto que, si bien hemos justificado que un sistema energético basado en fuentes renovables, intrínsecamente, no condiciona la geopolítica mundial, también se ha mostrado que sería posible diseñar territorialmente un sistema similar al anterior en el que, gracias al poder de monopolio de las empresas transnacionales que participaran en este, el nuevo modelo energético podría cumpliría con una función equivalente en el marco del sistema capitalista contemporáneo.

Desde este punto de vista, hay que preguntarse cuál será la sostenibilidad política y social de las energías renovables si se tiende hacia modelos energéticos como los aquí apuntados. Ante esta pregunta, es bueno recordar que, como se ha justificado, por las características de las renovables, el modelo energético que acabamos adoptando será el resultado de una elección política.

\section{Referencias bibliográficas}

JEVONS, S. W. (1906 [1865]). The Coal Question: and Inquiry Concerning the Progress of the Nation, and the Probable Exhaustion of Our Coal Mines. Londres: Macmillan and Co.

MALANIMA, P. (2014). «Energy in History». En: AGNOLLETI, M.; NERI SERNERI, S. (eds.) (2014). The Basic Enviromental History. Sulza: Springer International. DOI: https://doi.org/10.1007/978-3-319-09180-8_1

WARDE, P. (2013). «The First Industrial Revolution». En: KANDER, A.; MALANIMA, P.; WARDE, P. (eds.) (2013). Power to the People. Princeton: Princeton University Press.

WRIGLEY, E. A. (2010). Energy and the English Industrial Revolution [en línea]. Cambridge: Cambridge University Press. DOI: https://doi.org/10.1017/CBO9780511779619

Cita recomendada: MAÑÉ-ESTRADA, Aurèlia. La geopolítica de las renovables en el capitalismo del siglo XXI. Oikonomics [en línea]. Noviembre 2021, n. 16. ISSN: 2339-9546.

DOI: https://doi.org/10.7238/o.n16.2110 


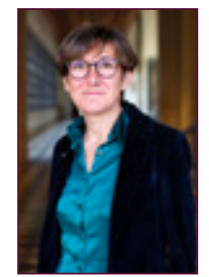

\section{Aurèlia Mañé-Estrada}

amimanera@ub.edu

\section{Universitat de Barcelona}

Doctora en Ciencias Económicas y Empresariales por la Universitat de Barcelona (UB) y máster en Relaciones Internacionales por la Universitat Autònoma de Barcelona (UAB). Es profesora titular en la UB, donde imparte asignaturas relacionadas con la política económica, la economía política de la energía y las economías del mundo árabe. Sus principales temas de investigación son las «economías petroleras» y las cuestiones energéticas, desde una perspectiva triple: la de la economía política, la geoenergética y la histórica-sistémica; entre sus publicaciones más recientes se encuentran los libros Argelia en transición hacia una segunda república (Icaria, 2019) y El gran negocio mundial de la energía (RBA, 2016). Por otro lado, ha colaborado con Casa Asia, donde creó y dirigió el Observatorio de Asia Central, y es miembro del grupo Economic History and Development (Industry, Business and Sustainability) de la Universitat de Barcelona, del grupo de energía y cambio climático del Real Instituto de Estudios Internacionales del Real Instituto Elcano y del Grupo de Estudio de las Sociedades Árabes y Musulmanas Contemporáneas (GRESAM, UCLM).

Los textos publicados en esta revista están sujetos -si no se indica lo contrario- a una licencia de Reconocimiento 4.0 Internacional de Creative Commons. Puede copiarlos, distribuirlos, comunicarlos públicamente, hacer obras derivadas siempre que reconozca los créditos de las obras (autoría, nombre de la revista, institución editora) de la manera especificada por los autores o por la revista. La licencia completa se puede consultar en https://creativecommons.org/licenses/by/4.0/deed.es_ES.

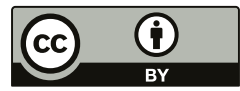

